\title{
Editorial
}

\section{Quality, the patient's charter, and primary care}

Family health services authorities (FHSAs) were required to develop and to publish charter standards and targets for their own internal services by 1 April $1993 .{ }^{1}$ In these charters they were asked to make explicit the existing rights of citizens as patients and to suggest how patients themselves can help primary care workers to deliver better services.

Another and much more difficult task charged to FHSAs is to encourage the development of charters in the setting of the family health services, starting with general practice. The Department of Health made it clear that general practice charters are voluntary; the role of FHSAs is to persuade practices that developing charters will be in their own and their patients' interests. Quality standards produced by the department, regional health authorities, or FHSAs and imposed on general practice will not work; ownership by the individual practices is vital if the charters are to function. The voluntary nature of these charters is likely to be a major strength and central to their success, but FHSA general managers must beware of simply allowing practices to produce platitudes purporting to be patient's charters that would merely be a paper exercise.

\section{Daunting task}

If FHSAs and other health authorities have found the subject of quality "managerially challenging" then many general practices have found the whole concept quite daunting. Developing practice standards may seem a simple and straightforward task, but it confronts some fundamental issues within a practice. For example, if a practice standard states that patients will be seen within 20 minutes of the appointment time, and all but one of the doctors in the partnership do, more or less, keep to time, the practice will be obliged by the process of producing the charter to discuss the one partner who has never kept to time. Charters expose these sorts of problems. Patients' charters are effective because they are shared not only within the practice but also with patients.

In Hereford and Worcester a scheme to develop the concept of quality within the setting of general practice has been running for two years. This "Pathfinder Initiative," originally aimed at four volunteer medical practices, has now reached $30 \%$ of general practices. By using feedback from patient surveys and outside facilitators each general practice focuses on issues of quality pertinent to it. Development of patients' charters is now included in this work.

An important issue emerging from this initiative is the interest and concern of consumers in local health service provision and the standard of primary care, which is not confined to special interest groups. Recently 2000 people from a partnership list of 10000 responded to an invitation to be shown around a new surgery, learn about the services offered, and meet the staff. Patient participation groups are an important part of charter and, indeed, practice development. Meeting regularly with the primary health care team, arranging meetings for patients, and finding mechanisms for incorporating consumer initiated suggestions are becoming part of practice culture. Newsletters are a way of involving and informing all those registered with a practice.

\section{First lessons}

What are the lessons so far from this venture into "charterland?" Firstly it is clear that this is only the start of introducing concepts of quality management into the setting of family health services. There is a long way to go in developing patient's charter standards and targets in general practice. It is a mistake to produce long, glossy, and expensive documents. Patients' charters should be dynamic and up to date, and they need to be reviewed every year. It is vital that they are part of the whole agenda of quality initiatives and not separate from other issues such as clinical standards. Perhaps the most important point is that the charters need to be genuinely "owned" by those who produce them and those whose care and service they are meant to improve. A final message for FHSAs and health authorities is to be certain that we deliver on our own quality standards. Otherwise our task of encouraging others to improve quality and meet their charter standards will be even more difficult.

General Manager,

CLIVE W PARR

Hereford and Worcester Family Health Services Authority,

Worcester WR1 2HP

1 NHS Management Executive. The patient's charter and primary health care. London: Department of Health, 1992. (EL(92)88.) 